
\title{
R Reserach S Suare \\ The Immune Cell Infiltration Patterns and \\ Characterization Score in Bladder Cancer to Identify Prognostic.
}

\section{Yongsheng Zhang}

Zhengzhou University

Yunlong Wang ( $\square$ biowyl@126.com)

Zhengzhou University https://orcid.org/0000-0002-9905-1769

Jichuang Wang

Institute of Bioengineering

Kaixiang Zhang

Zhengzhou University

\section{Research Article}

Keywords: Bladder cancer, common gene expression samples data, immune cell infiltration, prognosis

Posted Date: January 12th, 2022

DOI: https://doi.org/10.21203/rs.3.rs-1219557/v1

License: (c) (i) This work is licensed under a Creative Commons Attribution 4.0 International License.

Read Full License 


\section{Abstract}

Bladder cancer (BLCA) is among the most frequent types of cancer. Patients with BLCA have a significant recurrence rate and a poor post-surgery survival rate. Recent research has found a link between tumor immune cell infiltration (ICI) and the prognosis of BLCA patients. However, the ICI picture of BLCA remains unclear. Common gene expression data was obtained by combining the Cancer Genome Atlas (TCGA) and Gene Expression Omnibus (GEO) expression databases. Two computational algorithms were proposed to unravel the ICI landscape of BLCA patients. The R package "limma" was applied to find differentially expressed genes (DEGs). Principal-component analysis (PCA) was used to calculate the ICI score. A total of 569 common gene expression data were retrieved from TCGA and GEO cohorts. CD8+ T cells were found to have a substantial positive connection with activated memory CD4+ T cells and immune score. On the contrary, CD8+ T cells were found to have a substantial negative connection with Macrophages M0. Thirty-eight DEGs were selected. Two ICI patterns were defined by unsupervised clustering method. Patients of BLCA were separated into two groups. The high ICl score group exhibits better outcome than the low one $(p<0.001)$. Finally, the group with a high tumor mutation burden $(T M B)$ as well as a high ICl score had the best outcome. $(p<0.001)$. Combining TMB and ICl score resulted in a more accurate survival prediction, suggesting that $\mathrm{ICl}$ score could be used as a prognostic marker for BLCA patients.

\section{Introduction}

Bladder cancer (BLCA) is the world's tenth most prevalent cancer, accounting for around 549,000 new cases and 200,000 deaths in $2018^{1}$. As a highly heterogeneous tumor ${ }^{2-4}$, BLCA have a high recurrence rate (around 50\%) and the 5-year survival rate was around $60 \%$ after trimodally therapy ${ }^{5-7}$. Despite the rapid development of clinical imaging after chemotherapy and surgery, the method for evaluating therapeutic effect of BLCA is not satisfactory. As a result, developing new diagnostic, therapeutic, and prognostic biomarkers for BLCA is critical.

Due to their extraordinary and long-lasting anti-tumor effectiveness, Immune checkpoint inhibitors $\left(\mathrm{ICl}_{\mathrm{S}}\right)$ effectively changed the therapy of metastatic cancer ${ }^{8,9}$. Besides, $\mathrm{ICl}_{\mathrm{S}}$ are crucial in the inhibition of molecular receptor and ligand interactions, such as cytotoxic T-lymphocyte-associated protein 4 (CTLA-4) and programmed death-1 (PD-1) / programmed death ligand-1 (PD-L1) pathways, which are involved in initiating $T$ cell activation or function and rescuing immune monitoring ${ }^{10-11}$. However, the response of tumors to $\mathrm{ICl}_{\mathrm{S}}$ could hardly be analyzed ${ }^{12,13}$. TMB is a new biomarker for assessing $\mathrm{ICl}_{\mathrm{S}}$ efficacy, since it is associated with new antigens ${ }^{14-16}$. The breakpoint between TMB-high and TMB-low, like PD-L1, has yet to be determined ${ }^{17}$. Therefore, it is critical to find novel biomarkers that could predict the response of tumor to $\mathrm{ICl}_{S}$.

Extensive research has established the crucial involvement of $\mathrm{ICI}$ in cancer proliferation, recurrence, and metastasis $^{18,19}$. The higher the proportion of immune score in tumor microenvironment, the better 
prognosis in most of the patients ${ }^{20}$. Besides, Tumor-infiltrating lymphocytes (TLSs), including as CD4 and CD8 T cells, have been linked to increased survival rates ${ }^{21,22}$. In contrast, tumor-associated macrophages (TAMs) show poor prognosis by secreting immunosuppressive cytokines ${ }^{23-25}$. Nevertheless, recognizing TLS cells is insufficient to characterize the complicated tumor microenvironment. TAMs immunosuppressive cytokines may reduce TLSs' anti-tumor impact ${ }^{26}$. Additionally, increased stromal component filtration in tumor tissue might inhibit TLS transport to tumor, indicating that the link between the two sets of TME cells is more important than any single component ${ }^{7}$. In this study, two approaches "CIBERSORT" and "ESTIMATE" were employed to unveil the patient's ICI picture. CIBERSORT is mainly used to transform gene expression into the content of immune cells for each BLCA sample (filter conditions: $p<0.05$ ). The purpose of ESTIMATE is to obtain the score of immune cells and stromal components in BLCA samples. Besides, based on the $\mathrm{ICl}$ and $\mathrm{DEG}_{\mathrm{S}}, \mathrm{BLCA}$ patients were classified into two different subgroups. The $\mathrm{ICl}$ score was acquired by principal-component analysis (PCA). Finally, in this work, we developed the ICl score to describe distinct immune cell landscapes, which could predict exactly patient outcomes. As a result, we discovered that $\mathrm{ICl}$ score could serve as a prospective prognostic marker that is unrelated of TMB.

\section{Methods}

\section{BLCA Data collection}

TCGA and GEO databases were used to gather transcriptome and clinical data. In general, we collected two groups cohort samples of BLCA: GSE13507 and TCGA-BLCA. The exclusion criteria were as follows: (a) Not tumor tissue sample. (b) The transcriptome sequencing data or clinical information of the samples were incomplete. (c) Not common gene expression samples date. Finally, 569 samples were included. We converted the Fragments Per Kilobase Million (FPKM) values to the Transcripts Per Million (TPM) values by using the "limma" R package for TCGA-BLCA database. We combined TCGA and GEO expression date to get new common gene expression samples data for later analysis.

\section{The proportion of ICI was used to categorize BLCA patients.}

The "CIBERSORT" R package, the LM22 signature, and 1,000 permutations were used to evaluate infiltration levels for various immune cells in BLCA. ESTIMATE calculated the immune score and stromal score in BLCA patients. In addition, we acquired the correlation between different immune cells by using "corrplot" R package. The hierarchical agglomerative clustering of BLCA was implemented by different ICI patterns of each sample. The number of clusters were determined by consensus clustering algorithm. We performed the "ConsensusClusterPlus" R package and repeated 1000 times to ensure the stability of classification.

\section{Acquisition of differentially expressed genes $\left(D G_{S}\right)$ related to ICI Phenotype}


In order to find genes linked with ICl patterns, we classified patients into distinct groups based on ICl. DEGs among different groups were screened by means of the R package "limma". The significant criteria of $|\log \mathrm{FC}|>1$ and $\mathrm{p}$ (adjust) $<0.05$ were used to determine DEGs.

\section{Generation of ICI Score}

In order to further analysis, an unsupervised clustering method for DEG analysis was applied to divided the patients into different groups. Positive and negative DEG correlations with cluster signatures were classified as ICl gene signatures A and B, the "Boruta" algorithm was applied to reduce their dimensionality. Using the PCA, gene signature score of patients was derived. Finally, we used a procedure analogous to grading index gene expression to determine $\mathrm{ICl}$ score. ICl score $=\sum \mathrm{PC} 1 \mathrm{~A}-\sum \mathrm{PC} 1 \mathrm{~B}$.

\section{Collection of Somatic structural variation Data}

The correlation mutation information of patients in the TCGA-BLCA cohort was obtained from TCGA data portal (https://portal.gdc.cancer.gov/repository). In order to determine tumor mutation burden, we calculated the total number of non-synonymous mutations in BLCA. We got 20 driver genes through the $\mathrm{R}$ package "maftool", which had the highest mutation frequency in BLCA patients. Finally, we evaluated whether differences in the mutation frequency of genes between two ICl score groups.

\section{Gene Ontology (GO) and Gene Set Enrichment Analysis (GSEA)}

The "clusterprofiler" package was utilized for gene annotation and enrichment analysis of ICI distinctive genes. GO terms were screened by a stringent cut off $(P<0.05)$. Futuremore, we used a GSEA to find pathways that were up and down regulated between two ICl score groups. The parameter settings were Gene sets database = "Kyoto Encyclopedia of Genes and Genomes (KEGG)", n Perm = 1000 and $P<0.05$.

\section{Analysis of Clinical Features in two ICI score groups}

The corresponding clinical data from the TCGA and GEO databases was retrieved and manually organized. We verified predictive value of ICI score with distinct Clinical features (such as age and gender) by the R package" survival".

\section{Statistical analysis}

All data is analyzed by $\mathrm{R}$ software (version 4.0.4). The proportion of 22 types immune cells in BLCA was calculated by "CIBERSORT" algorithm. The "ConsensusClusterPlus" R package was used to divide BLCA patients into two types. DEGs between two ICI Phenotype are filtered through R package "limma". The ICI score was calculated by PCA algorithm. Further analysis, TMB was obtained in TCGA-BLCA by "TMB.pl". The prognosis of BLCA patients was evaluated by R-package" survival". The "clusterprofiler" package was used for gene annotation and enrichment analysis of $\mathrm{ICl}$ distinctive genes. The predictive value of $\mathrm{ICI}$ scores with different clinical characteristics (such as age and gender) was verified by R-package "survival". $P<0.05$, considered statistically significant. 


\section{Results}

\section{The Pattern of ICl in the TME of BLCA}

The workflow is displayed in Fig. 1. Firstly, a total 569 common gene expression data were extracted from TCGA and GEO cohorts. Then, the CIBERSORT and ESTIMATE algorithms were applied to assess the level of immune cells (Filter conditions: $p<0.05$ ) in BLCA patients. (Supplementary Table $1 \& 2$ ). The correlation coefficient heatmap shows that the significant positive correlation of CD8 T cells with activated memory CD4 T cells and immune score. On the contrary, the significant negative correlation of CD8 T cells with Macrophages M0 (Fig. $3 \mathrm{C}$ ). Based on results of immune cell infiltration, BLCA patients were divided into two different ICl subtypes by R package "conesusclusterplus" unsupervised clustering. The consensus matrix was the crispest when $\mathrm{K}=2$ (Fig. 2A-2D), namely ICl cluster A and B (Supplementary Table 3). The heatmap enables visualization of the amounts of expression of immune cells of distinct ICl clusters (Fig. 3B). Moreover, two independent ICl subtypes showed significant difference in the overall survival rate $(p=0.002$; Fig. $3 A)$.

To better explain and comprehend the biological and clinical distinctions among these inherent features, we analyzed the immune cell composition of two ICl subtypes. Between two ICl subtypes, ICl cluster A had a better outcome with a median duration of roughly 5 years. Meanwhile, it was marked by increased infiltration CD8 T cells, activated memory CD4 T cells and resting Mast cells, ect. In addition, the ICI cluster A has higher immune score than the ICl cluster B. On the contrary, the ICl cluster B confirmed a poor prognosis (median survival duration roughly 3 years) and performed a large rise in the amount of Macrophages M0 (Fig. 3D).

\section{Identified the subtypes of immune related gene}

To understand the underlying biological properties of distinct immunophenotypes, we used R package "limma" to carry out differential analysis to identify the transcriptome differences between two subtypes. To determine the DEGs, unsupervised clustering was implemented by the "limma" package (Supplementary Table 4). We classified BLCA patients into gene clusters A-B by DEGs (Fig. 4A-4D; Supplementary Table 5). The positive correlation of DEGs values with the clusters signature, were coded as $\mathrm{ICl}$ gene signature $\mathrm{A}$, while the remaining $\mathrm{DEG}$ s were coded as $\mathrm{ICl}$ gene signature $\mathrm{B}$. At the same time, to remove interference or duplicated genes, we employed the "Boruta" method to minimize the dimensionality of gene signatures $A$ and $B$. The transcriptome properties of DEGs are shown in a heat map created with the R package "pheatmap." The R package "clusterProfiler" was applied to execute GO enrichment analysis on the signature genes. Fig. 5B-5E illustrates the biological processes that have been significantly enriched, and Supplementary Table 6 contains a thorough explanation.

Next, we evaluated the prognosis of gene clusters A-B combined with survival information. It showed that two independent gene clusters had significant difference in overall survival ( $p<0.001$; Fig. $5 F)$. The gene cluster A was characterized with a better outcome (median survival duration roughly 5.5 years), 
whereas the gene cluster B was linked to a poor outcome. (median survival duration roughly 2 years). As displayed in Fig. 5G, the gene cluster A showed obvious increase in the infiltration of some immune cells, like as CD8 T cells and naive B cells. Meanwhile, the gene cluster B performed a higher Macrophages M0 infiltration. Finally, some differentially expressed target genes were analyzed in two gene clusters by the "limma" package.

\section{Generation of ICI Score}

In develop a quantification of the ICl environment in BLCA patients, the PCA algorithm was applied to generate two overall scores: the $\mathrm{ICl}$ score $A$ from $\mathrm{ICl}$ signature gene $A$ and the $\mathrm{ICI}$ score $B$ from $\mathrm{ICl}$ signature gene $\mathrm{B}$. We obtained the sum of individual scores using $\mathrm{ICl}$ scores $\mathrm{A}$ and $\mathrm{B}$ of each sample in the study. Finally, we obtained the ICI score, which is a predictive signature score. The TCGA-BLCA and GSE13507 patients were separated into high and low ICl score groups using "survival" package (Supplementary Table 7). The alluvial diagram described the correlation among the gene clusters, the ICI score and survival outcomes (Fig. 6A). CD274, CTLA4, HAVCR2, LAG3, and PDCD1 were chosen as immune-checkpoint-relevant signatures, and CD8A, CXCL10, CXCL9, GZMA, GZMB, IFNG, PRF1, TBX2, and TNF were selected as immune-activity-related signatures, to investigate the immunological activation and tolerant state of the TCGA-BLAC and GSE13507 cohorts. With the exception of TBX2, the ICI score was shown to have a substantial negative correlation with the expression quantity of immunecheckpoint-relevant and immune-activity-relevant genes. (Fig. 6B). Moreover, GSEA analysis results revealed that fatty acid metabolism and PPAR signaling pathways were considerably enriched in the high $\mathrm{ICl}$ score group, whereas Proteasome and NOD-like receptor signaling pathways were substantially enriched in the low one (Fig. 6C). A detailed enrich information description was provided in Supplementary Table 8.

Next, we investigated at how the ICl score affected a patient's prognosis. It showed that two independent $\mathrm{ICl}$ score groups had remarkably difference in the overall survival rate $(\mathrm{p}<0.001 ; \mathrm{Fig} .6 \mathrm{D})$. The high ICl score group showed a good prognosis (median survival duration roughly 5.3 years), whereas the low one had the unfavorable outcome (median survival duration roughly 1.2 years).

Lastly, "ggplot2" package was applied to evaluate the relation between ICl score and survival status. We found that two independent ICI score groups had significant difference in survival status. The majority of BLCA in high ICl score group were alive, on the contrary, the majority of BLCA in low one were dead $(p=$ 0.0059; Fig. 6E \& F).

\section{TMB \& ICI score were applied to assess the prognosis of TCGA-BCLA cohort patients}

Since BLCA was reported to have high degree of somatic changes, subsequently, we determined the distribution of somatic mutations and combined with $\mathrm{ICl}$ score to evaluate the prognosis of patients. Firstly, the total mutation burden and mutation distribution of TCGA-BCLA were obtained by analyzing mutation annotation files. Meanwhile, we sorted the patients into high and low TMB groups. As 
demonstrated in Fig. 7A, we discovered that high TMB group was related to better outcome than the low one $(p<0.001)$. Considering the contraindication value of TMB and ICI score for prognosis, we subsequently studied the synergistic effect of $\mathrm{ICl}$ score in the prognostic classification of BLCA. The results reveal that there was a substantial difference in survival between the high and low TMB groups depending on $\mathrm{ICl}$ score subtypes. Among them, the high TMB combined with high ICl score had the best outcomes in BLCA ( $p<0.001$; Fig. 7B). In conclusion, The ICl score could be utilized as a possible predictor irrespective of TMB, which could effectively predict the response of immunotherapy.

In addition, twenty driver genes with the greatest mutation frequency were chosen for research development. We evaluated at the allocation of driver genes in two ICl score groups. The result showed that the alteration frequency of TP53, KMT2D, PIK3CA, KMT2C and FLG was considerably different between two ICl score groups (Fig. 7C-7D). Moreover, we discovered that the TP53 mutation frequency was higher in low $\mathrm{ICl}$ score group. The result proved once again that, the group with the low $\mathrm{ICI}$ had a poor prognosis. These results may provide new ideas for the study of the mechanism of $\mathrm{ICl}$ in tumor.

\section{Analysis of Clinical traits in two ICl score groups}

To be able to clarify the function of ICI score in BLCA, the relationship between ICI score and clinical characteristics was researched. The stratified survival analysis was used to observe whether ICl score could be applied to different clinicopathological features. Next, we analyzed patients' age and gender. Results showed that ICI score could effectively forecast OS in all groups from the age and gender clinical characteristics (Fig. 8A-8D).

\section{Discussion}

At the moment, the primary therapeutic strategy for localized BLCA is radical resection, next to intracavitary chemotherapy or immunotherapy ${ }^{28}$. However, BLCA has the characteristic of high recurrence rate and low survival rate ${ }^{5-7}$. Despite the fact that ICls are effective against advanced urothelial malignancies, including BLCA, tumor reaction to ICls is often poor and difficult to predict. ${ }^{12,13}$. Besides, TMB is considered as an important marker predicting $\mathrm{ICl}$ response in a variety of tumor types. Nevertheless, the boundary between high and low TMB has yet to be properly defined ${ }^{17}$. As a result, finding a novel prognostic marker is critical.

Instead of tumor cells, more and more attention has been paid to $\mathrm{ICl}$ recently. In this research, we combined TCGA-BLCA and GSE13507 to get common gene expression data, which contains 569 BLCA patient samples. Subsequently, based on unsupervised clustering method, a total 569 patient samples were divided into two different immune subtypes according to the proportion of $\mathrm{ICl}$. Consensus clustering has been widely used in genome research ${ }^{29}$. Based on the DEGs between $\mathrm{ICl}$ cluster $\mathrm{A}$ and ICl cluster $\mathrm{B}$, we classified the BLCA patients into two genomic clusters, gene cluster A and B. Anti-tumor cells and protumor cells are two kinds of immune cells engaged in cancer local immune response ${ }^{30,31}$. Different immune cells could conduct distinct functions in different tumors ${ }^{32}$. Our analysis results showed that the 
expression levels of CD8 T cells and naive B cells were up-regulated in gene cluster A, indicating a good outcome. Meanwhile, Macrophages M0 were shown to be positively related with gene cluster $\mathrm{B}$, indicating a bad outcome.

We obtained two gene signatures by different expression levels of DEGs in different gene clusters. Given the individual variability of the immunological milieu, quantifying the $\mathrm{ICI}$ model for individual tumors is critical $^{33}$. In some cancers, individual-based models have been fully established to improve outcome forecasting ${ }^{34,35}$. In this study, the PCA algorithm was used to separate the TCGA-BLCA and GSE13507 cohorts into two $\mathrm{ICl}$ score groups. The high score group has a better prognosis than the low one. Through GSEA, we found that the genes implicated in immune activation pathway, such as fatty acid metabolism and PPAR signaling pathways, were significantly abundant in the high $\mathrm{ICl}$ score group. In various $\mathrm{ICl}$ score groups, we analyzed the levels of immune activation related signal and immune checkpoint related signal. In low ICI score group, the expression levels of CD274, CTLA4, HAVCR2, LAG3, PDCD1, CD8A, CXCL10, CXCL9, GZMA, GZMB, IFNG, PRF1 and TNF were up-regulated, except TBX2. Besides, we explored the mutation frequency of some driving genes in different $\mathrm{ICl}$ score groups. The results revealed that in the low ICl score group, the frequency of TP53 mutations was increased. All of these studies revealed that the $\mathrm{ICl}$ score was adversely linked with tumor malignancy from different perspectives. Since the neoantigen load could be easily detected and evaluated by TMB, it has been proved to be an indicator of clinical benefit and a prognostic factor for predicting $\mathrm{ICl}$ response. Our analysis shows that high TMB has better OS performance in BLCA. Finally, we found that the high TMB combined with something like the high $\mathrm{ICl}$ score has higher survival rate than others. ICI score can effectively predict the OS of age and gender groups. However, all results of this study were obtained retrospectively based on public databases, which requires further prospective validation.

\section{Conclusions}

We carefully investigated the BLCA ICl environment, resulting in a comprehensive view of anti-/pro-tumor immune response modulation in BLCA. The variation of $\mathrm{ICI}$ patterns is related to tumor heterogeneity. As a result, this discovery has significant clinical implications for the comprehensive examination of tumor

$\mathrm{ICI}$ patterns. Our results revealed that $\mathrm{ICl}$ score could be served as an efficient predictive marker which is independent of TMB. These findings could contribute to a novel strategy to predict the prognosis of BLCA.

\section{Declarations}

\section{Author Contributions}

Yongsheng Zhang conceived the article, conducted statistical analysis and wrote the manuscript. Yunlong Wang conceived and reviewed the article. Jichuang Wang reviewed the article and data analysis. Kaixiang Zhang revised the manuscript. 


\section{Funding}

The research was supported by National Natural Science Foundation of China (U19042).

\section{Data availability}

The RNA-sequencing profles were extracted from TCGA and GEO databases, which were open-access.

\section{Code availability}

R software (version 4.0.4) was used for analysis and plotting.

\section{Conflict of interest}

The authors declare no competing interests.

\section{References}

1. Bray F, Ferlay J, Soerjomataram I, Siegel RL, Torre LA, Jemal A. Global cancer statistics 2018: GLOBOCAN estimates of incidence and mortality worldwide for 36 cancers in 185 countries. CA: a cancer journal for clinicians. 2018;68(6):394-424.

2. Knowles MA, Hurst CD. Molecular biology of bladder cancer: new insights into pathogenesis and clinical diversity. Nature reviews. Cancer. 2015;15(1):25-41.

3. Babjuk M, Böhle A, Burger M et al. EAU Guidelines on Non-Muscle-invasive Urothelial Carcinoma of the Bladder: Update 2016. European urology, 2017;71(3):447-461.

4. Li P, Cao J, Li J et al. Identification of prognostic biomarkers associated with stromal cell infiltration in muscle-invasive bladder cancer by bioinformatics analyses. Cancer medicine. 2020;9(19): 72537267.

5. Cambier S, Sylvester RJ, Collette L, et al. EORTC Nomograms and Risk Groups for Predicting Recurrence, Progression, and Disease-specific and Overall Survival in Non-Muscle-invasive Stage TaT1 Urothelial Bladder Cancer Patients Treated with 1-3 Years of Maintenance Bacillus CalmetteGuérin. European urology. 2016;69(6): e123-4.

6. Kamat AM, Hahn NM, Efstathiou JA, et al. Bladder cancer. Lancet 2016;388: 2796-2810.

7. Sanli O, Dobruch J, Knowles MA et al. Bladder cancer. Nature reviews. Disease primers. 2017;3: 17022.

8. Johnson DB, Sullivan RJ, Menzies AM et al. Immune checkpoint inhibitors in challenging populations. Cancer. 2017;123(11):1904-1911.

9. D'Aiello A, Lin J, Gucalp R et al. Thyroid Dysfunction in Lung Cancer Patients Treated with Immune Checkpoint Inhibitors (ICls): Outcomes in a Multiethnic Urban Cohort. Cancers, 2021,13(6).

10. Topalian SL, Drake CG, Pardoll DM et al. Immune checkpoint blockade: a common denominator approach to cancer therapy. Cancer cell. 2015,27 (4):450-61. 
11. Tøndell A, Subbannayya Y, Wahl SGF et al. Analysis of Intra-Tumoral Macrophages and T Cells in Non-Small Cell Lung Cancer (NSCLC) Indicates a Role for Immune Checkpoint and CD200-CD200R Interactions. Cancers. 2021;13 (8).

12. Zou W, Wolchok JD, Chen L et al. PD-L1 (B7-H1) and PD-1 pathway blockade for cancer therapy: Mechanisms, response biomarkers, and combinations. Science translational medicine. 2016;8(328):328rv4.

13. Dermani FK, Samadi P, Rahmani G et al. PD-1/PD-L1 immune checkpoint: Potential target for cancer therapy. Journal of cellular physiology. 2019;234(2):1313-1325.

14. Rizvi NA, Hellmann MD, Snyder A et al. Cancer immunology. Mutational landscape determines sensitivity to PD-1 blockade in non-small cell lung cancer. Science (New York, N.Y.). 2015;348(6230): 124-8.

15. Hellmann MD, Callahan MK, Awad MM et al. Tumor Mutational Burden and Efficacy of Nivolumab Monotherapy and in Combination with Ipilimumab in Small-Cell Lung Cancer. Cancer cell. 2019;35(2): 329.

16. Zhao DY, Sun XZ, Yao SK. Mining The Cancer Genome Atlas database for tumor mutation burden and its clinical implications in gastric cancer. World journal of gastrointestinal oncology. 2021;13(1): 37-57.

17. Samstein RM, Lee $\mathrm{CH}$, Shoushtari AN et al. Tumor mutational load predicts survival after immunotherapy across multiple cancer types. Nature genetics. 2019;51(2):202-206.

18. Zeng $D$, Zhou $R, Y u$ Y et al. Gene expression profiles for a prognostic immunoscore in gastric cancer. The British journal of surgery. 2018;105(10):1338-1348.

19. Jiang Y, Zhang Q, Hu Y et al. ImmunoScore Signature: A Prognostic and Predictive Tool in Gastric Cancer. Annals of surgery. 2018;267(3):504-513.

20. Na KJ, Choi H. Immune landscape of papillary thyroid cancer and immunotherapeutic implications. Endocrine-related cancer. 2018;25(5):523-531.

21. Vassilakopoulou M, Avgeris M, Velcheti V et al. Evaluation of PD-L1 Expression and Associated Tumor-Infiltrating Lymphocytes in Laryngeal Squamous Cell Carcinoma. Clinical cancer research: an official journal of the American Association for Cancer Research. 2016;22(3):704-13.

22. Zeng D, Li M, Zhou R et al. Tumor Microenvironment Characterization in Gastric Cancer Identifies Prognostic and Immunotherapeutically Relevant Gene Signatures. Cancer immunology research. 2019;7(5):737-750.

23. Noy R, Pollard JW. Tumor-associated macrophages: from mechanisms to therapy. Immunity. 2014;41(1):49-61.

24. Chen $Y P$, Wang YQ, Lv JW et al. Identification and validation of novel microenvironment-based immune molecular subgroups of head and neck squamous cell carcinoma: implications for immunotherapy. Annals of oncology: official journal of the European Society for Medical Oncology. 2019;30(1):68-75. 
25. Liang B,Tao Y, Wang T. Profiles of immune cell infiltration in head and neck squamous carcinoma. Bioscience reports. 2020;40(2).

26. Şenbabaoğlu Y, Gejman RS, Winer AG et al. Tumor immune microenvironment characterization in clear cell renal cell carcinoma identifies prognostic and immunotherapeutically relevant messenger RNA signatures. Genome biology. 2016;17(1):231.

27. Chen DS, Mellman I. Elements of cancer immunity and the cancer-immune set point. Nature. 2017;541(7637):321-330.

28. Cao R, Yuan L, Ma B, Wang G, Tian Y. Immune-related long non-coding RNA signature identified prognosis and immunotherapeutic efficiency in bladder cancer (BLCA). Cancer cell international. 2020; 20:276.

29. Șenbabaoğlu Y, Michailidis G, Li JZ. Critical limitations of consensus clustering in class discovery. Scientific reports. 2014; 4:6207.

30. Riera-Domingo C, Audigé A, Granja S et al. Immunity, Hypoxia, and Metabolism-the Ménage à Trois of Cancer: Implications for Immunotherapy. Physiological reviews. 2020;100(1):1-102.

31. Yu YR, Ho PC. Sculpting tumor microenvironment with immune system: from immunometabolism to immunoediting. Clinical and experimental immunology. 2019;197(2):153-160.

32. Foley K, Kim V, Jaffee E, Zheng L. Current progress in immunotherapy for pancreatic cancer. Cancer letters. 2016;381(1):244-51.

33. Callari M, Cappelletti V, D'Aiuto F et al. Subtype-Specific Metagene-Based Prediction of Outcome after Neoadjuvant and Adjuvant Treatment in Breast Cancer. Clinical cancer research: an official journal of the American Association for Cancer Research. 2016;22(2):337-45.

34. Bramsen JB, Rasmussen MH, Ongen $\mathrm{H}$ et al. Molecular-Subtype-Specific Biomarkers Improve Prediction of Prognosis in Colorectal Cancer. Cell reports. 2017;19(6):1268-1280.

35. Komor MA, Bosch LJ, Bounova $\mathrm{G}$ et al. Consensus molecular subtype classification of colorectal adenomas. The Journal of pathology. 2018;246(3):266-276.

\section{Figures}


Exploratory analysis

A total of 686 BLCA samples datasets
$($ TCGA-BLCA $n=430)($ GSE13507 $n=256)$

Delete not common gene expression tumor samples

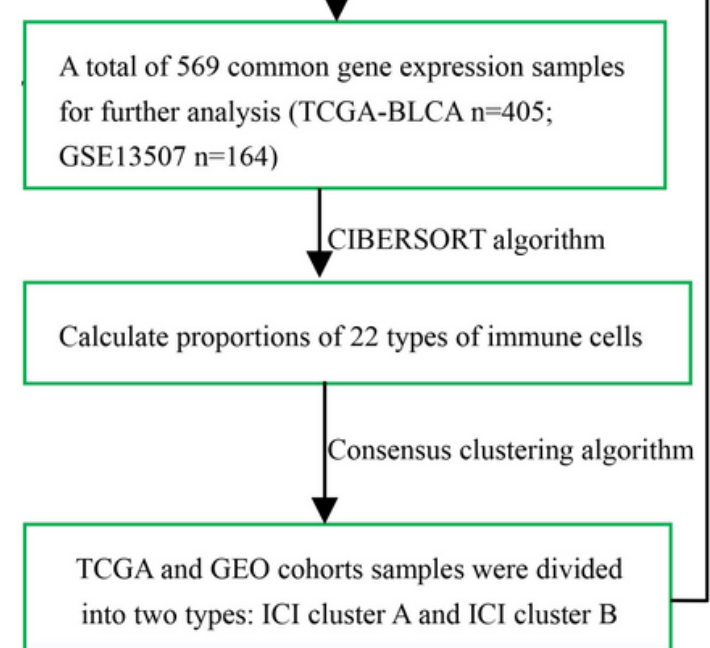

Discovery study

Determine DEGs between two ICI clusters

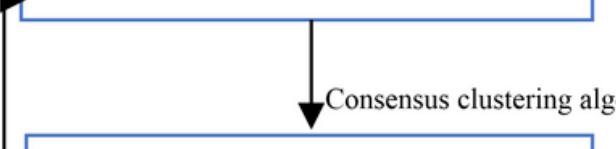

Patients in TCGA and GEO cohorts were classified into two groups: gene cluster A and gene cluster $B$
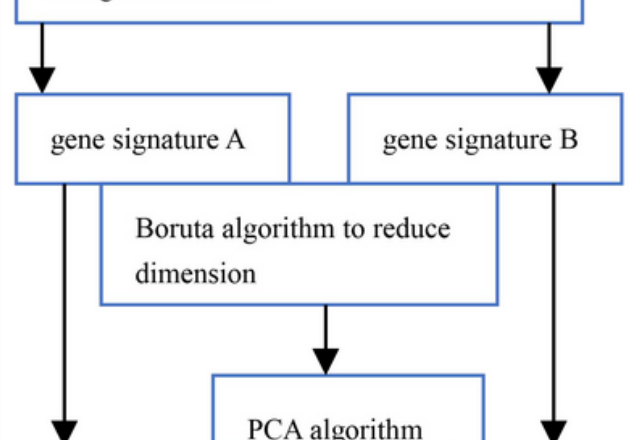

ICI score B
Further analysis

Somatic structural variation Data from

TCGA data portal

$\int^{\text {TMB.pl }}$

MB was obtained in TCGA-BLCA



\section{Figure 1}

Legend not included with this version. 
A

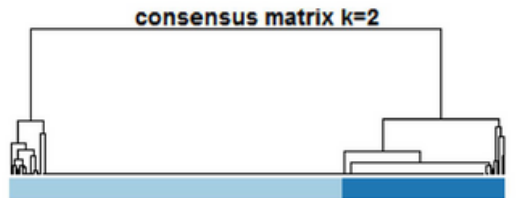

\begin{tabular}{|l|}
\hline$ㅁ$ \\
$\square$ \\
\hline
\end{tabular}

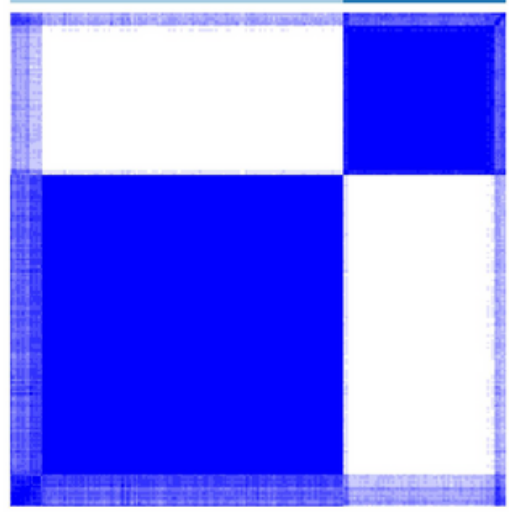

C

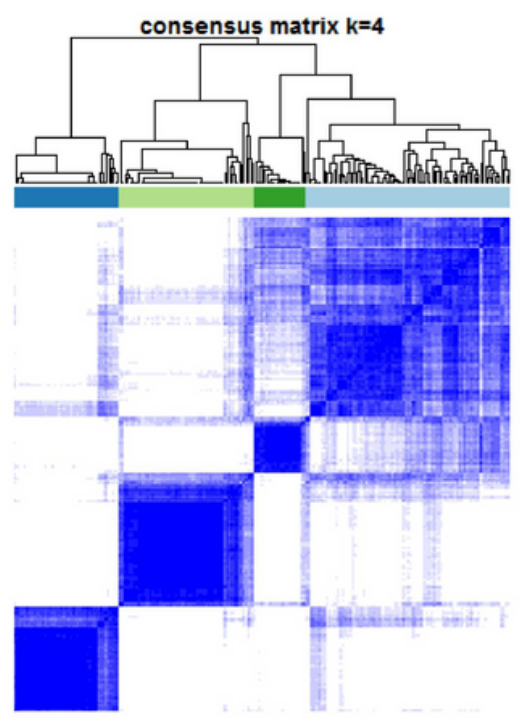

B

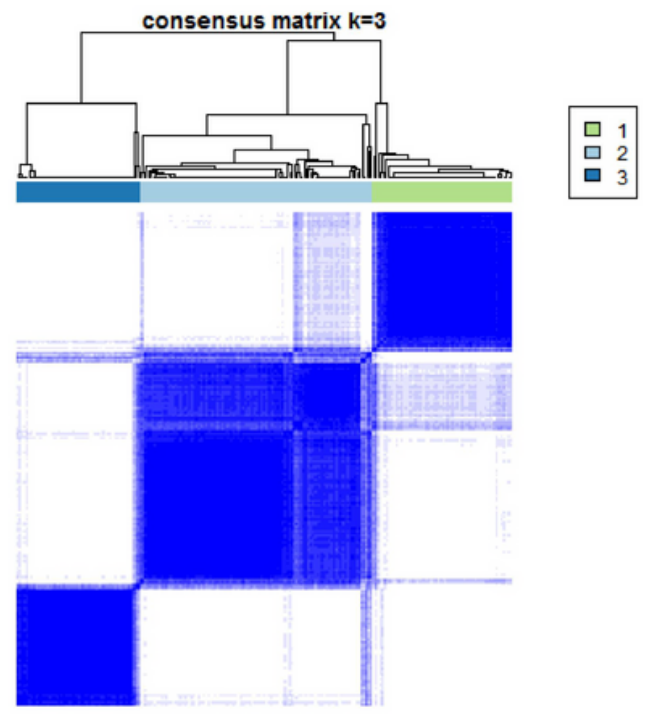

D

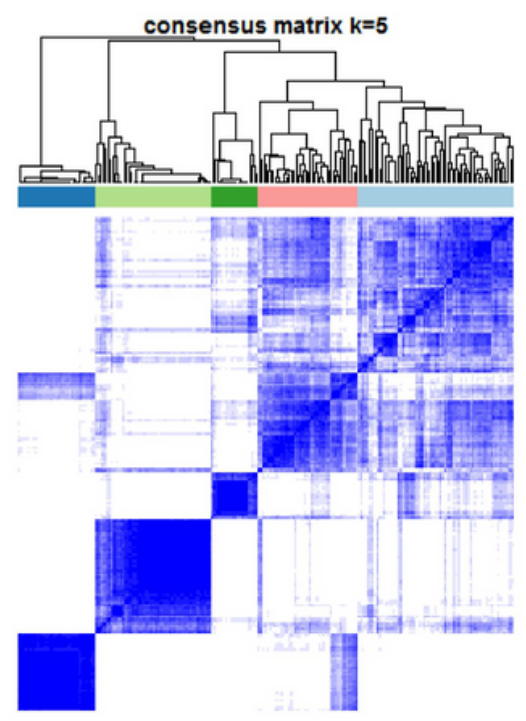

Figure 2

Legend not included with this version. 
A



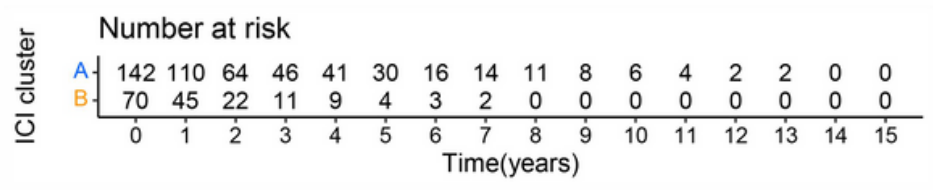

C

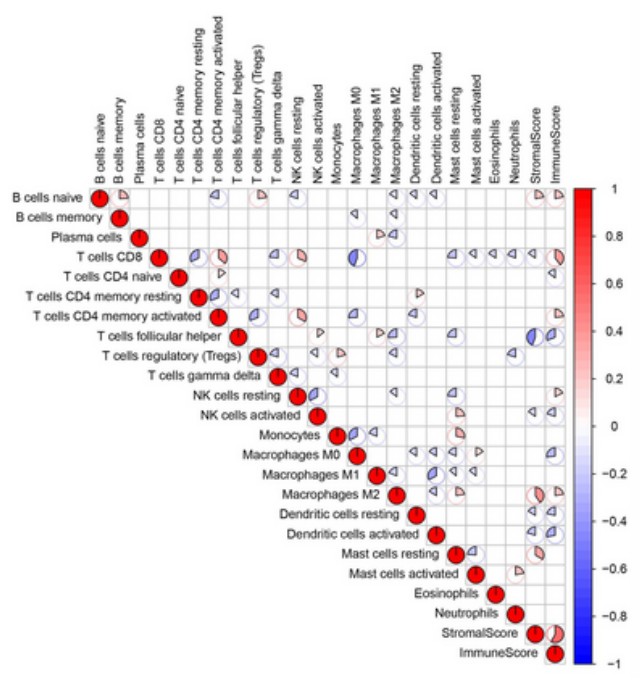

B

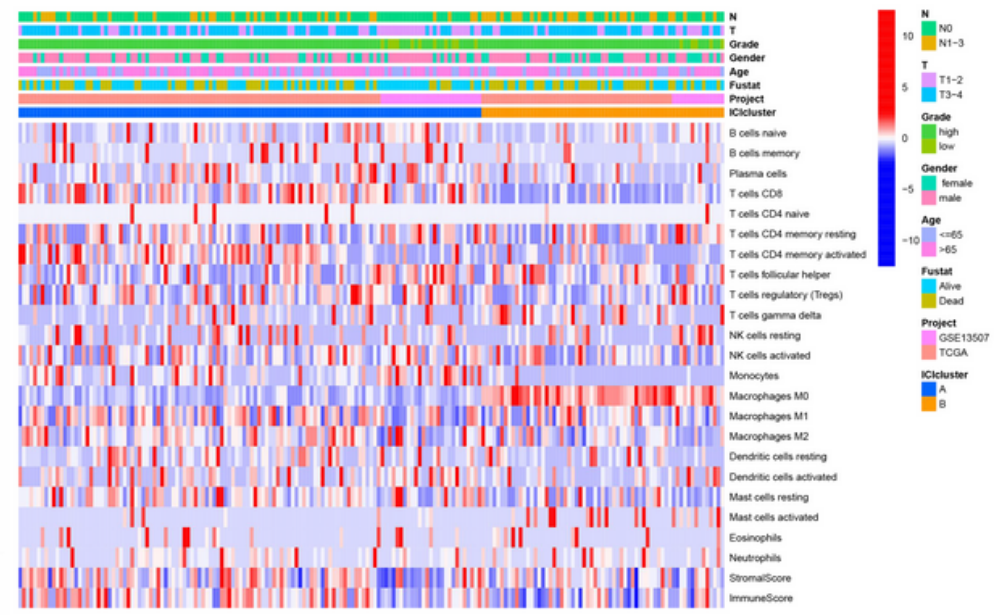

D

ICl cluster 官 A 官 $\mathrm{B}$

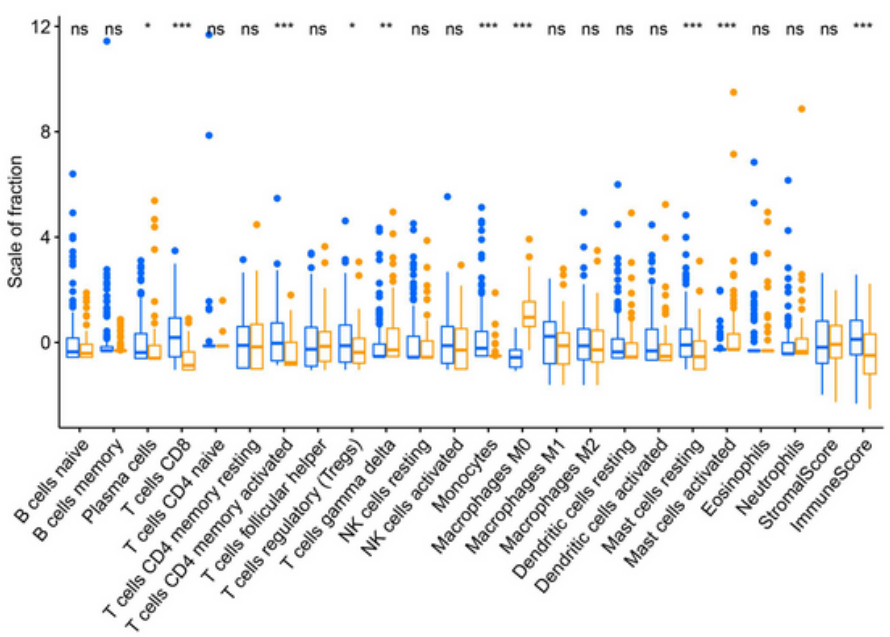

Figure 3

Legend not included with this version. 
A

B



\begin{tabular}{ll}
\hline \\
$\square$ \\
\hline
\end{tabular}

C



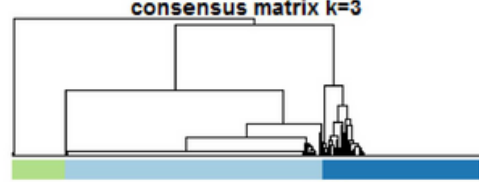

\begin{tabular}{ll} 
믐 2 \\
․ 3 \\
\hline
\end{tabular}

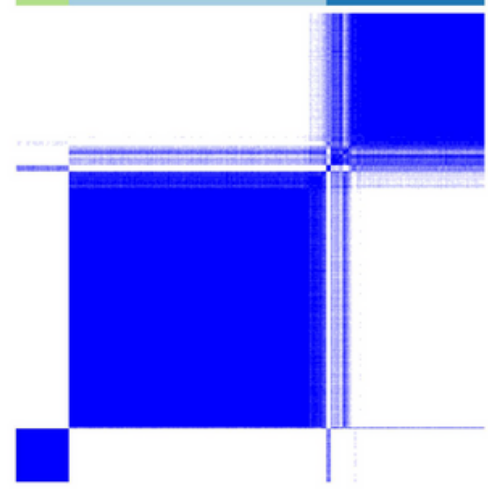

D

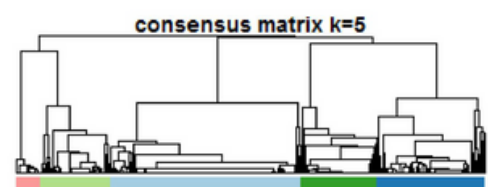

Figure 4

Legend not included with this version. 
A

C
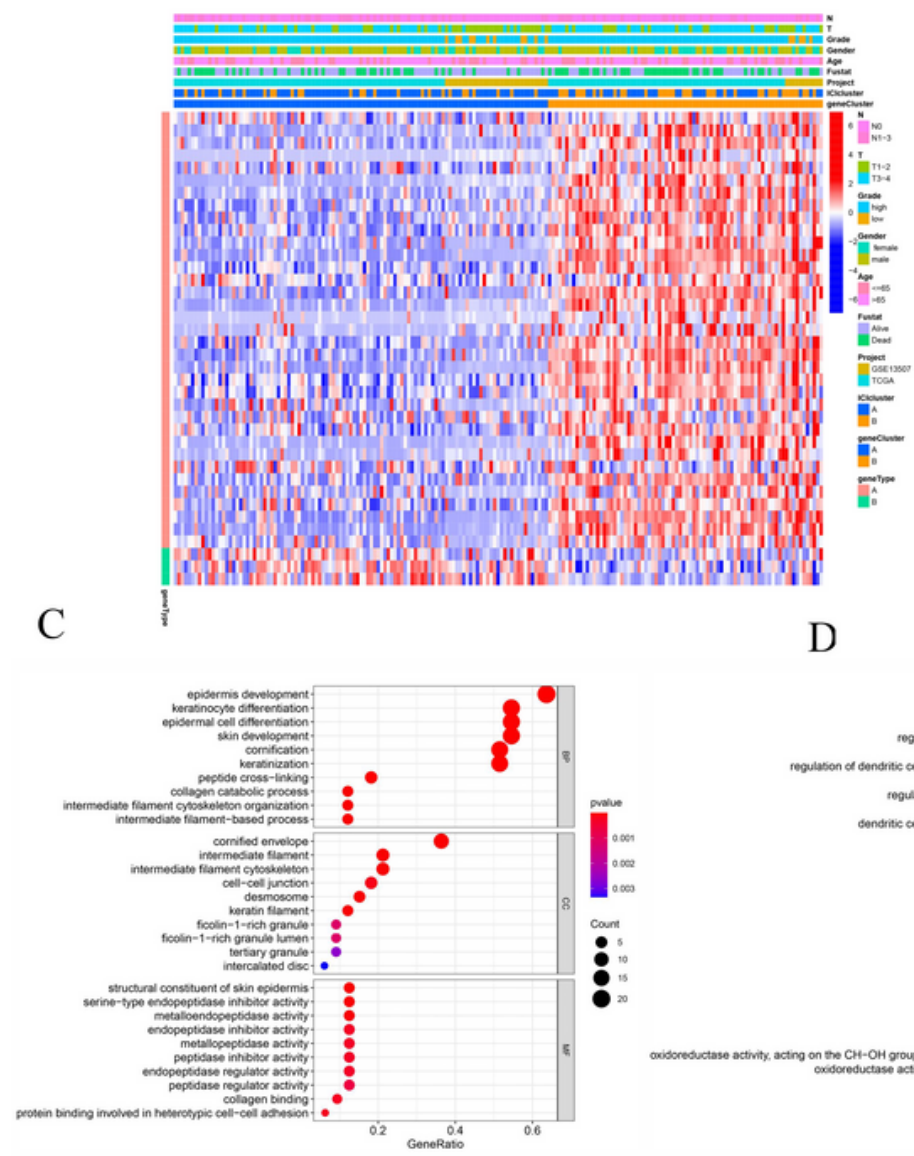

$\mathrm{F}$

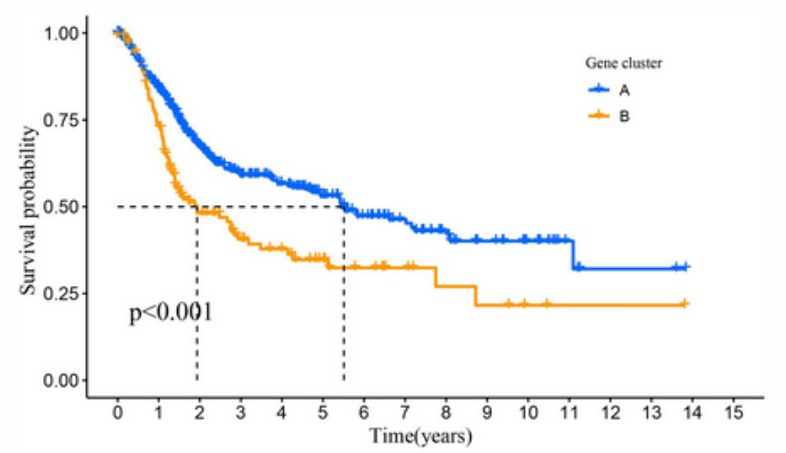

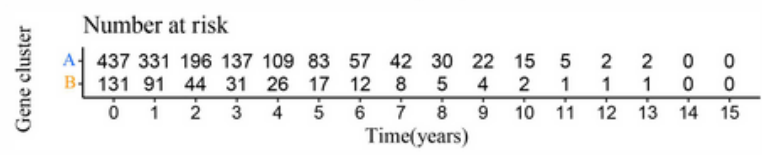

B

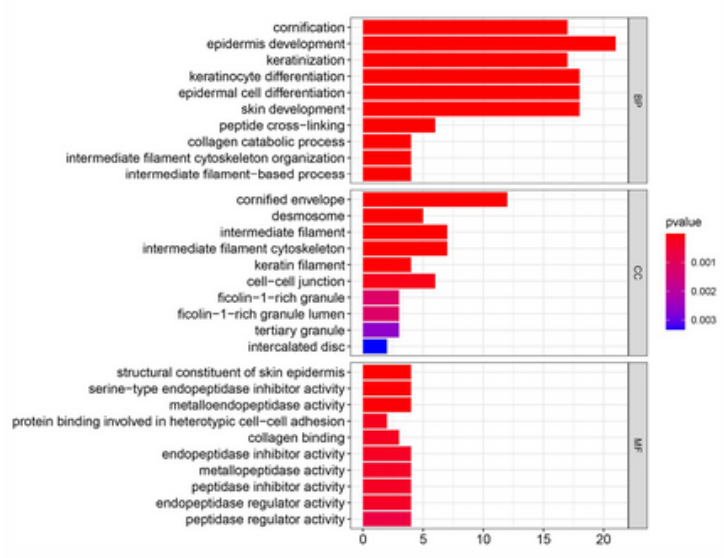

E

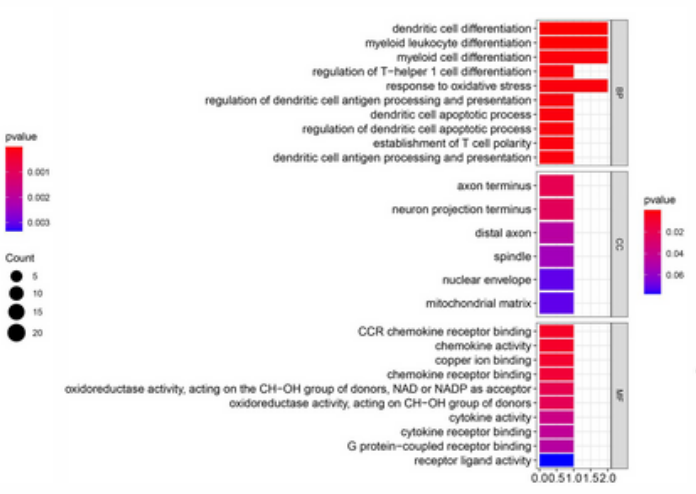

G
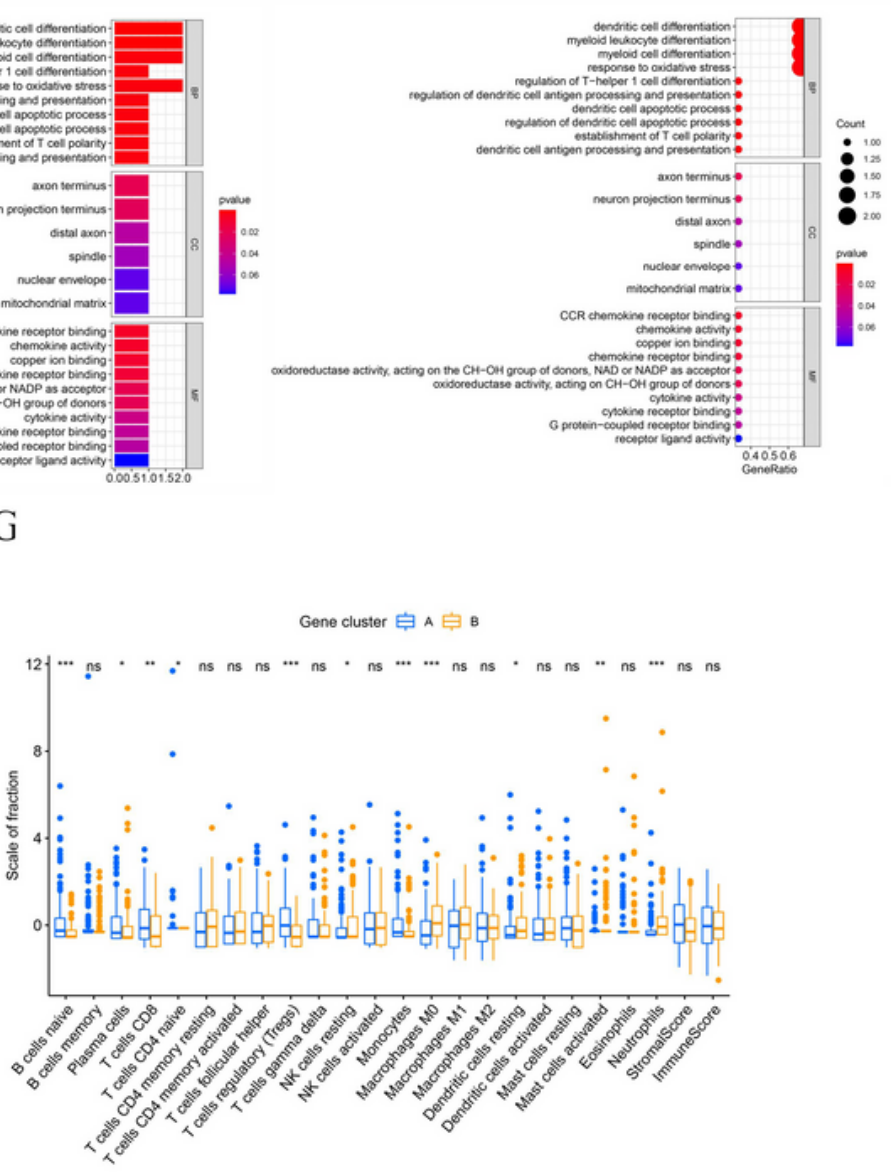

\section{Figure 5}

Legend not included with this version. 
A



$\mathrm{C}$
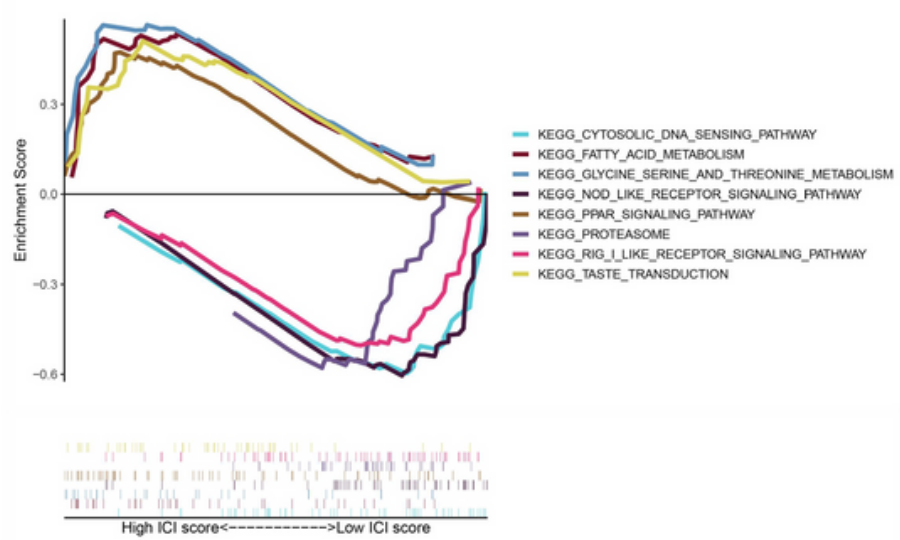

E



B

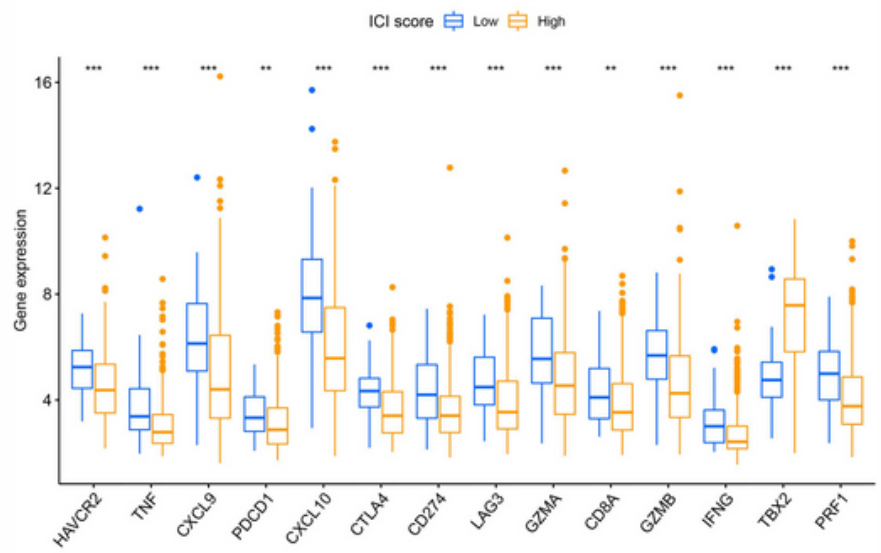

D

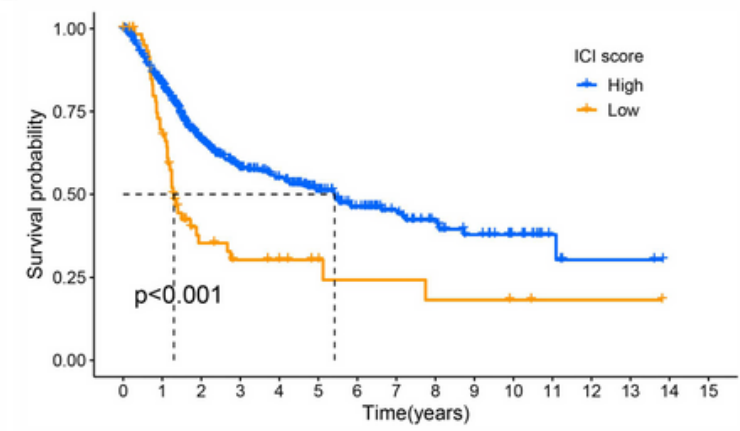

Number at risk

总 High. $\begin{array}{cccccccccccccccc}505 & 381 & 225 & 157 & 125 & 94 & 65 & 46 & 32 & 23 & 15 & 5 & 2 & 2 & 0 & 0 \\ 63 & 41 & 15 & 11 & 10 & 6 & 4 & 4 & 3 & 3 & 2 & 1 & 1 & 1 & 0 & 0\end{array}$

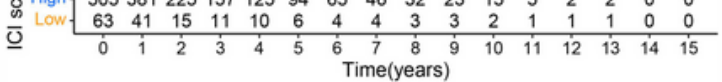
F

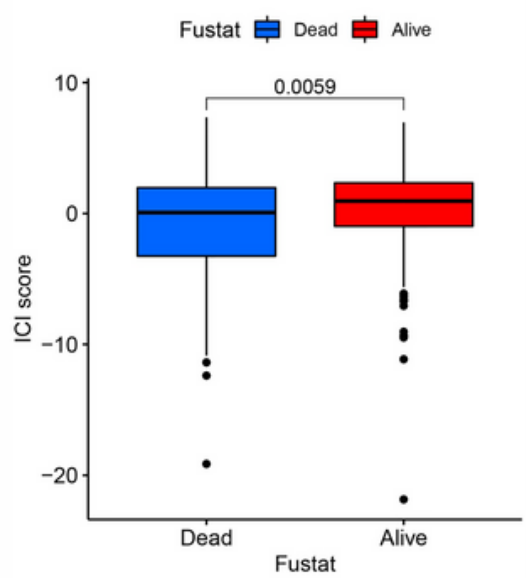

Figure 6

Legend not included with this version. 
A



Number at risk



C

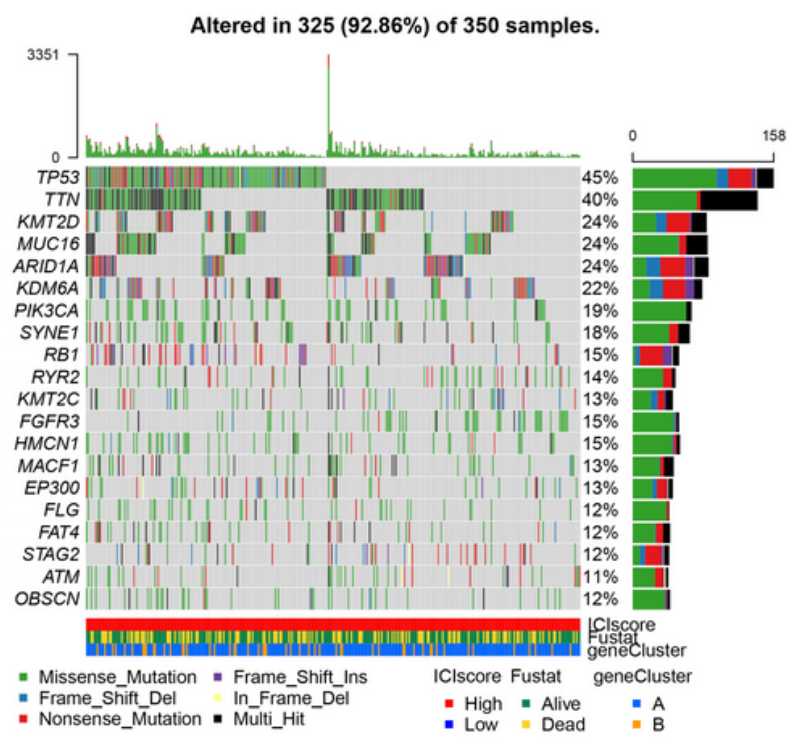

B

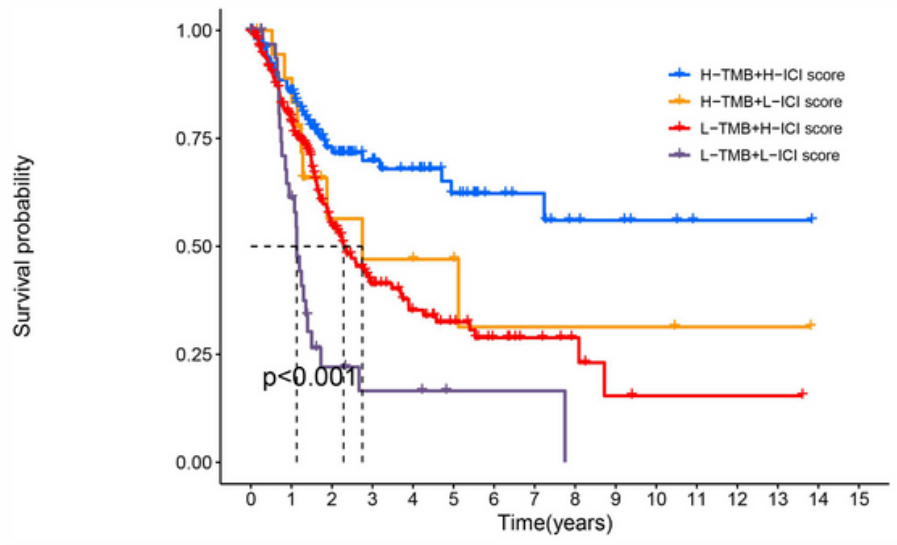

Number at risk



D

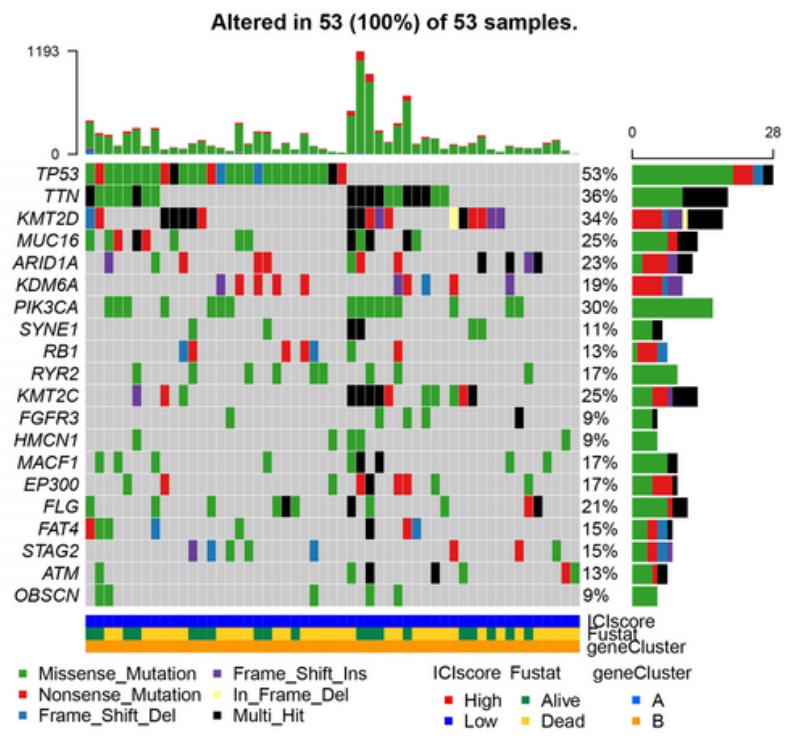

Figure 7

Legend not included with this version.

Figure 8

Legend not included with this version.

\section{Supplementary Files}

This is a list of supplementary files associated with this preprint. Click to download. 
- SupplementaryTable1.xls

- SupplementaryTable2.xls

- SupplementaryTable3.xls

- SupplementaryTable4.xls

- SupplementaryTable5.xls

- SupplementaryTable6.xls

- SupplementaryTable7.xls

- SupplementaryTable8.xls 